\title{
Metagenomic Analysis Reveals Associations between Salivary Microbiota and Body Composition in Early Childhood
}

Modupe Coker ( $\nabla$ moyegunle@gmail.com )

Rutgers University https://orcid.org/0000-0002-9072-7953

Rebecca Lebeaux

Geisel School of Medicine at Dartmouth https://orcid.org/0000-0002-3046-1225

Anne Hoen

Dartmouth College

Yuka Moroishi

Geisel School of Medicine at Dartmouth

Diane Gilbert-Diamond

Geisel School of Medicine at Dartmouth

\section{Erika Dade}

Geisel School of Medicine at Dartmouth

\section{Thomas Palys}

Geisel School of Medicine at Dartmouth

Juliette Madan

Dartmouth Geisel School of Medicine

\section{Margaret Karagas}

Dartmouth College

\section{Article}

Keywords: saliva microbiome, microbiota, obesity, children, body mass index

Posted Date: September 28th, 2021

DOI: https://doi.org/10.21203/rs.3.rs-909959/v1

License: (c) (i) This work is licensed under a Creative Commons Attribution 4.0 International License.

Read Full License 


\section{Metagenomic Analysis Reveals Associations between Salivary Microbiota and Body \\ 2 Composition in Early Childhood}

3 KEYWORDS: saliva microbiome; microbiota; obesity; children; body mass index

Several studies have shown that body mass index is strongly associated with differences in gut 8 microbiota, but the relationship between body weight and oral microbiota is less clear. Among

9 more than 200 toddlers in the New Hampshire Birth Cohort Study, we characterized the

10 association between multiple anthropometric measures of body mass/growth longitudinally and 11 used shotgun metagenomics to taxonomically and functionally profile the oral microbiome. We 12 found that within-sample diversity was inversely related to body mass measurements while 13 community composition was not associated. Certain taxa were consistently associated with 14 growth and modified by sex. Functional examination also showed concordance between 15 microbial metabolic pathways and child growth metrics. Further exploration of the functional 16 significance of this relationship will enhance our understanding of the intersection between 17 weight gain, microbiota, and energy metabolism and the potential role of these relationships on 18 the onset of obesity-associated diseases in later life. 
Introduction

2 Obesity in young children is associated with premature death and disability in adulthood. In the

3 United States, the prevalence of obesity among children aged $2-5$ years increased from $5 \%$ in

41980 to over $13 \%$ in 2018 [1], making it a significant and growing public health problem.

5 Overwhelming evidence from animal and human studies suggests that the gut microbiome

6 influences the risk of overweight and obesity [2-4]. Several studies (mostly in animals and

7 several in humans) observed differences of important bacterial species in the gut microbiota

8 between obese and normal weight/lean subjects, with some studies showing a higher

9 Firmicutes/Bacteroidetes (F/B) ratio in obese/overweight subjects compared to those of normal

10 weight $[5,6]$. Data also suggest that lifestyle alterations and physical activity in turn alter

11 the gut microbiome, and that these changes are dependent on obesity status [3, 7]. The

12 mechanisms posited to underly these relationships are increased energy harvest, regulation of

13 host metabolism, and the activation of innate immunity.

14 The relationship between the oral bacteriome and obesity is less clear with emerging

15 evidence suggesting that dysbiosis of the oral microbiome is related to the underlying

16 imbalances and metabolic processes leading to the acquisition of body fat/weight [8, 9]. Beyond

17 the well-known orodental diseases like caries, gingivitis and periodontitis, the oral microbiome is

18 associated with systemic inflammation and increased risk for health outcomes including

19 cardiovascular disease, diabetes, rheumatoid arthritis and inflammatory bowel disease [10, 11].

20 As the start of the alimentary canal and home to volumes of saliva ingested daily, the oral cavity

21 has the potential to provide microbial information about the gastrointestinal tract with bacteria

22 regularly passing through the oral cavity to the gut. As observed in the gut, an altered oral

23 microbiome has been associated with metabolic changes and obesity [12-14] in both

24 adolescents and adults [15]. However, there is limited data on the relationship between obesity

25 and the oral microbiota in early childhood. 
In a previous study that compared adults and adolescents with obesity vs. normal

27 weight, body mass index (BMI) was shown to differ significantly with respect to the proportion of

28 Campylobacter rectus and Neisseria mucosa, as well as Tannerella forsythia in the subgingival

29 biofilm with greater abundance [13]. Taxa within the genera Bifidobacterium, specifically $B$.

30 longum, and Lactobacillus in saliva were cross-sectionally associated with lower obesity

31 prevalence, lower BMI, and lower weight gain. These differences in the microbial composition

32 indicate that there may be distinct patterns of association between the salivary microbiome and 33 obesity.

34 The oral microbiome is crucial to a child's oral and systemic health through its role in

35 immune training and seeding of the infant gut [16]. Based on existing literature, we hypothesize

36 that salivary microbiota in children would vary in diversity and richness by age, growth scores,

37 adiposity and fat mass. Our focus on early childhood is based on documented changes with complete primary dentition, suggesting establishment of a complex oral microbiome that likely lays a foundation of health with increasing age $[17,18]$, highlighting the critical need to characterize factors that contribute to the development of the microbiome in early life. Equally

41 important is the likelihood that the oral microbiota mirrors the maturation and stability of the gut 42 microbial community by age 3 observed by several groups [19-21]. High BMI in preschool years 43 (and not later in childhood) has been shown to be associated with a higher risk of overweight or 44 obesity in adolescence among children who had had stable BMI [22]. Therefore, in examining 45 the salivary microbiota of approximately 4-year-old children enrolled in an ongoing prospective 46 cohort, we aimed to investigate whether the salivary microbiota was associated with concurrent 47 body weight metrics (overweight status or body fat mass). For a subset of participating children, 48 we examined the potential impact of early growth exposure metrics prior to sample collection 49 (weight trajectory up to age 2) on the salivary microbiota. 
Results

\section{Characteristics of study population}

54 Out of 273 children enrolled in NHBCS with saliva microbiome samples available, we focused

55 on 236 that had weight, height, and dual-energy x-ray absorptiometry (DXA) measurements

56 collected at the same time. Of these, the average age of participants was 1410 days (81.9 SD)

57 or almost 4 years old. The distribution of descriptive characteristics for study participants by

58 overweight status is shown in Table 1 . The majority $(165 ; 69.9 \%)$ of participants were at a

59 normal weight while $62(26.3 \%)$ were classified as overweight or obese. As depicted in Table

60 S1, among the 236 children, 131 (55.5\%) were male. To incorporate prospective anthropometric

61 growth data prior to the time of saliva sample collection, we created 2 exploratory sub-cohorts

62 from the 273 children (Figure S1A) with 137 children conserved in all 3 cohorts (Figure S1B). A

63 total of 195 children had weight-for-length/height ratios available (Table S2). Rapid weight gain

64 (RWG), a commonly used dichotomous child growth metric that typically is defined as an

65 increase in weight-for-age z-score $>0.67$ between birth and a 2-year weight measurement [23,

66 24], was assessed in 157 children with available data in the first 2 years of life. Among them, 45

67 (28.7\%) were classified as having RWG (Table S3).

69 grams. Ultimately, five variables were selected to assess body composition for children at 3-4

70 years of age: 1) TFM as reported from DXA; 2) TLM as reported from DXA; 3) age- and sex-

71 adjusted BMI z-score; 4) age- and sex-adjusted BMI percentiles to categorize children as

72 underweight $\left(<5^{\text {th }}\right)$, healthy weight $\left(5^{\text {th }}\right.$ to $\left.<85^{\text {th }}\right)$, overweight $\left(85^{\text {th }}\right.$ to $\left.<95^{\text {th }}\right)$, or obese $\left(\geq 95^{\text {th }}\right)$; and

73 5) a binary variable for overweight with the age- and sex-adjusted BMI $85^{\text {th }}$ percentile used as

74 the threshold.

75 Relationships between child growth measurements

76 Body mass index and DXA-measured TFM were highly but not perfectly correlated

77 (Pearson correlation, $r=0.80)$ (Figure S2A), whereas DXA-measured lean mass was only 
moderately correlated with BMI $(r=0.47)$ (Figure S2B). Likewise, in linear regression models,

79 adjusted for age and sex, a unit BMI increase was associated with a 777 (95\% Cl: 705,848$)$

80 gram increase in TFM and a $499(95 \% \mathrm{Cl}: 392,607)$ gram increase in TLM respectively. As

81 expected, male and female children had different mean anthropometric measurements with

82 male children being taller (Kruskal-Wallis $p<0.05)$, heavier $(p<0.05)$, and with a higher amount of

83 TLM ( $p<0.05)$. Using our exploratory sub-cohorts, we validated that both rapid weight gain

84 between 0 and 2 years and weight-for-length ratio were associated with BMI at 3-4 years of age

85 among both males and females (Figure 1A and Figure 1B).

\section{Characterization of salivary microbiota and association with growth metrics}

A total of 627 GB of raw data was generated from the Illumina NextSeq platform. After

filtering out low-quality data and host contamination, an average of 22.9 million reads of clean data were retained for each sample. The majority of saliva microbiota was made up of

91 Firmicutes followed by Proteobacteria, Actinobacteria, and Bacteroidetes (Figure 2A). Across

92 the 236 children, 203 species and 54 genera were identified in at least $1 \%$ of samples. The top

93 genera by mean relative abundance (Figure 2B) were Streptococcus, Gemella, and Neisseria

94 and the top species (Figure 2C) were Streptococcus mitis, Gemella haemolysans, and Rothia 95 mucilaginosa. showed a moderately inverse association with BMI z-scores (Table 2). Although TFM was associated negatively with alpha diversity in a crude/unadjusted analysis, TLM was not

100 associated at all. Between sample or beta diversity analysis using principal coordinate analysis

101 (PCoA) plots showed moderate associations with TFM and BMI Z-scores (Figure S3). Within

102 PERMANOVA models, TFM and BMI z-scores were statistically significant (Table S4 and Table 
103 S5; crude model $p$-values $<0.05$; adjusted model $p$-values $<0.1$ ) with microbial beta diversity 104 but described very little of either model's variation.

105 Using MaAsLin2, we explored associations between child growth metrics and individual 106 saliva microbiota (Table S6). Although we found limited results reaching statistical significance 107 (Benjamini-Hochberg q-value < 0.25), we observed a high level of concordance in the direction 108 of associations for taxa across metrics assessed. Of the top 10 genera by $p$-value in adjusted 109 models for TFM and BMI z-score at 3 or 4 years of age, 7 genera overlapped (Figure 3A). Of

110 those 7, Granulicatella and Streptococcus abundance were positively associated while 111 Actinomyces, Neisseria, Prevotella, Rothia, and Veillonella were negatively associated. 112 Regarding species, six species overlapped between growth measurements with Actinomyces 113 odontolyticus and Prevotella melaninogenica abundance consistent across all three models 114 (Figure 3B). To further demonstrate the consistency and effect size similarities between 115 models, we subsequently ran adjusted univariate linear regression models on specific taxa and 116 plotted the overlap between species (Figure 3C). Across all these species with a low p-value 117 via MaAsLin2, the effect estimates across all child growth metrics were consistent.

118 Due to inherent differences in child growth by sex, we were interested in assessing the 119 joint interaction of sex and child growth on the saliva microbiota. At the genus level, we found 120 evidence of an antagonistic joint effect of female sex and child growth on Streptococcus while 121 assessing both age and sex-adjusted BMI z-score as well as DXA-measured fat mass (Figure 122 4A) At the species-level, we found consistent results to the genus-level analysis. A synergistic 123 joint effect of female sex and child growth on Neisseria cinerea was also observed (Figure 4B). 124 For males, an antagonistic joint effect with child growth was noted for Neisseria and Neisseria 125 cinerea (Figure 4C; Figure 4D).

126 Finally, we aimed to better understand how child growth might be associated to 127 functional differences in the saliva microbiome. While results did not indicate strong 128 associations between child growth metrics and KEGG gene families or pathway abundances, 
129 we found consistently positive associations across models assessing TFM and age and sex-

130 adjusted BMI z-scores. Among functional pathways, we identified sugar and methionine

131 pathways including lactose and galactose degradation I and L-methionine biosynthesis I (Figure

132 5A, Figure S4A, and Figure S4B). Within KEGG gene families, we found congruent hits for

133 multiple gene families related to large subunit ribosomal proteins as well as for energy-coupling

134 factor transport system ATP-binding proteins (Figure 5B, Figure S4C, and Figure S4D).

\section{Associations between other early-life factors and the saliva microbiome}

As this study is one of the first to profile oral microbiomes with shotgun metagenomics in

138 children 3-4 years of age, we performed exploratory analyses of the impact of other early-life

139 factors on saliva microbiota. Regarding diversity metrics, we found maternal BMI was positively

140 associated with Shannon alpha diversity in all models. Maternal BMI, age of saliva sample

141 collection, and sex were moderately associated with beta diversity but no variables explained a

142 significant portion of the variance. Additionally, in our differential abundance analyses using

143 MaAsLin2, we consistently identified maternal BMI to be positively associated with Veillonella

144 parvula and Veillonella. Independently, increasing age of the child and female sex were also

145 associated with increased abundance of Haemophilus. While other associations between early-

146 life exposures and microbes were often of similar direction and magnitude across models, no

147 other early-life exposures were found to be statistically significant (Benjamini-Hochberg q-value $148<0.25)$.

150 Discussion

151 In the present study, we characterized the oral microbiome using shotgun sequencing

152 technology and investigated its relationship with age- and sex-adjusted BMI and body

153 composition. Our study is among the first to provide comprehensive metagenomic insight into

154 the association between growth outcomes and the salivary microbiome in early childhood. 
155 While there were no strong taxa-specific associations, we identified multiple bacterial taxa

156 (including Actinomyces, Corynebacterium, Capnocytophaga, Prevotella, Streptococcus mitis

157 and Veillonella) to be moderately associated with TFM, a child's overweight status, BMI and

158 RWG. Further, we identified high levels of concordance for these taxa with respect to

159 abundance and direction across the various anthropometric measurements. Our study

160 highlights the potential interactions between child growth and sex, with an antagonistic

161 interaction noted for Streptoccocus abundance among females but a synergistic interaction with

162 Neisseria cinerea. Overall, we found that various taxa within the phylum Firmicutes,

163 Actinobacteria, Bacteroidetes were associated with body composition and weight gain in the first 164 two years of life.

165 Bacterial- and host-genome-association studies of obesity are complex, multifactorial

166 and bidirectional in nature. The wide range of host and environmental effects and the significant

167 inter-individual variability of the oral microbiome makes interpretation of studies, such as ours,

168 challenging. Furthermore, in examining the association between oral microbiota and obesity in

169 pre-school children, the accurate assessment of growth and adiposity is critical. We observed

170 that BMI Z-scores were more highly correlated with DXA-derived fat mass compared to lean

171 mass, confirming earlier reports and providing validation to the anthropometric measurements

$172[25]$.

173 Literature provides strong evidence of significant differences in the human gut

174 microbiome comparing people with obesity to controls [2, 4]. There is some consensus of

175 increased levels of gut Firmicutes to the detriment of Bacteroidetes [26] with obesity and type 2

176 diabetes. Early-life gut microbiota is strongly influenced by dietary factors including the

177 introduction of formula and solid food $[21,27,28]$. The most dominant and differentially

178 abundant taxa in the infant gut due to obesity was Firmicutes followed by Bacteroidetes [29], as

179 has also been found in adult studies [30]. It has been hypothesized that having higher gut

180 levels of Firmicutes promotes more efficient storage of energy from a given diet among obese 
181 subjects compared with lean subjects. Although gut studies focus on fecal bacteria, all bacteria

182 from the gastrointestinal tract must pass through the oral cavity and are potentially seeded from

183 the oral cavity [31]. The relationship between the oral microbiota composition and obesity is less

184 clear as there have been mixed and inconsistent results. This is primarily due to variation in

185 study population, methodology, body weight assessments and microbiome characterization. Our

186 study is among the first to utilize WGS, included more than 100 participants or focus exclusively

187 on pre-school children. Previous studies have reported no differences in oral microbiota

188 composition according to BMI $[32,33]$ while others have observed distinct features [34-36].

189 Nevertheless, there is growing evidence of a significant association between levels of specific

190 oral bacterial taxa and obesity, BMI and weight gain $[35,37,38]$. This increase in attention

191 stems from the relationship between body weight and oral health, specifically the manifestation

192 of periodontitis, gingivitis and dental caries. These findings lead to a logical thread of

193 investigations related to answering the question "Is the relationship between obesity and oral

194 health mediated by the oral microbiome?".

195 Our findings of lower diversity in the oral microbiome with increasing BMI and fat mass

196 are in line with previous studies [39]. In contrast to our findings, several studies observed no

197 difference [32] while one study reported a higher diversity in obese children [40]. We observed

198 no clear clustering of beta diversity indices by BMI or DXA measurements. This finding is likely

199 due to the large inter-individual variation in the salivary microbiome among healthy-weight

200 children leading to considerable overlap in distance measures as observed by others [32]. Our

201 study findings highlight lower levels of Prevotella (from phylum Bacteroidetes) in overweight

202 children. Goodson et al [41] reported that oral Prevotella spp. was more abundant in overweight

203 women compared to normal weight women. In contrast, no significant association between

204 oral Bacteroidetes with obesity was observed in a large study of African-American adults aged

$205>50$ [35]. Prevotella species dominate in periodontal diseases and abscesses and are often

206 associated with mucosal inflammation [42]. Prevotella in the gut has been previously shown to 
207 be negatively associated with BMI and fat mass in children [43] as we observed with saliva.

208 Other studies of adolescents and adults have identified Prevotella to be positively associated

209 with aging and pro-inflammatory cytokines [44], which is consistent with findings that obesity is

210 associated with low-grade inflammation. Overall, these conflicting findings signal the need for

211 future work.

212 The relationship between periodontal disease and obesity has also drawn more attention

213 to the role of the oral microbiota in obesity. Adult studies have shown that obesity is associated

214 with increased counts and proportions of certain periodontal pathogens, including Tannerella

215 forsythia and Selenomonas noxia [45]. Our study population offers an advantage of examining

216 the association between oral microbiota and obesity in childhood as children are not typically at

217 risk of inflammatory diseases or conditions associated with aging such as periodontitis, diabetes

218 and cardiovascular disease therefore the associations with obesity can be the focus of

219 investigation [46]. Consistent with this premise, and as expected, we did not observe any

220 significant associations between body weight and well known pathobionts. S. mitis, considered

221 one of the beneficial commensal bacteria and an emerging opportunistic pathogen when in

222 niches distal to the oral cavity, was observed as being among the most taxonomically abundant

223 and functionally active species with respect to anthropometric measures. Its contribution to

224 childhood growth and weight/fat gain therefore requires further examination.

225 RWG in early childhood has been identified as a risk factor for obesity in adolescence

226 and adulthood and its associated complications [47]. We report differentially abundant taxa

227 based on RWG that seemed to overlap with other growth measures. However, we identified two

228 overlapping taxa were associated in opposing directions for RWG compared to concurrent BMI

$229 \mathrm{Z}$ scores. It is not clear if these differences are due to distinct periods of childhood growth.

230 Furthermore, RWG in first 2 years of life was not observed in all children who were overweight

231 at approximately 4 year of age, suggesting that RWG in early life does not always reflect the

232 same growth patterns later in life. Craig et al [37] sequenced hypervariable regions V3 and V4 
233 of the 16S rRNA gene in oral and stool samples from over 200 two-year-olds and utilized

234 functional data analysis to examine childhood weight-gain trajectories longitudinally. The

235 authors report that in children who gained weight rapidly from birth to six months of age, oral

236 bacterial diversity at two years of age was decreased with a higher Firmicutes to Bacteroidetes

237 ratio; but this was not observed with the gut microbiota [37]. While within-sample diversity and

238 F/B ratio are key summary tools for assessing of the microbiota, they are limited in their ability to

239 identify obesity-related features of the fecal or salivary microbiota. Nevertheless, the findings

240 from Craig and colleagues [37] suggest that obesity-related associations may appear at an

241 earlier time point for saliva microbiota than in the gut microbiota. Future investigations may hold

242 promise of leveraging the oral microbiome as a biomarker for health outcomes in relationship to

243 the gut microbome.

244 There are several mechanisms by which weight gain could contribute to the oral

245 microbiota or vice versa. Many postulate that bacteria in the oral cavity could contribute to

246 systemic metabolic alterations, as with gut Firmicutes. Specific oral taxa could contribute to

247 redirecting consumption of energy by facilitating insulin resistance through increasing levels of

248 TNFa and lipo-polysaccharides or reducing levels of adiponectin. In addition, oral microbiome

249 could also contribute to taste perception [40] and appetite control.

250 While our findings were related to the oral cavity, the association between maternal BMI

251 and Veillonella parvula in the gut has been previously reported by Costa and colleagues [48]. It

252 is plausible to consider maternal BMI a proxy variable for child's diet [49]. In addition to the role

253 of V. parvula in association with weight gain, costimulatory properties of Streptococcus and

254 Veillonella spp. have been observed by several investigators in-vitro and across various human

255 microbial ecosystems $[50,51]$. Specifically, some streptococci when combined with Veillonella

256 substantially augmented immune cell profiles including IL-8, IL-6, IL-10, and TNF- $\alpha$ responses.

257 These data suggest similar interactions and require further investigation particularly in the oral

258 milieu where Streptococcus is the predominant genera. 
A strength of this study is utilization of high-resolution whole genome sequence data to

260 characterize the oral microbiota. To our knowledge, there is no previous study that has applied

261 shotgun sequencing to saliva samples collected in early childhood for the purpose of this

262 evaluation. Use of 165 data by previous studies lend to poor resolution of oral microbial taxa.

263 Additionally, we were also able to leverage DXA measurements of body composition. To our

264 knowledge, the relationship between DXA-measured fat mass and salivary microbiota has not

265 been explored in children. Despite these strengths, our findings need to be examined in light of

266 several limitations. The cross-sectional design of this study has its inherent weakness; however,

267 for a subset we were able to address the potential impact of early life growth on development of

268 the oral microbiome. We also recognize that there is the potential for unmeasured or residual

269 confounding based on unexplored associations or due to the use of covariates with dampened

270 effects due to previous, as opposed to, current exposure [37]. Specifically, as discussed earlier,

271 although the impact of diet on the association between weight gain/growth and the

272 establishment of the oral microbiota was not directly assessed in this study, we considered

273 maternal BMI as an alternative indicator and adjusted for it in all our analyses.

274 In conclusion, our data from 3 to 4-year-old children suggested a lower diversity with

275 increasing BMI and body composition and highlights some differences in oral microbial

276 composition on the basis of BMI (based on overweight status) and TFM. These findings suggest

277 that changes in the body composition might impact the oral microbiome in early childhood or

278 vice versa, further increasing the risk of disease in later life. A larger sample size and

279 prospective follow-up will help determine whether the observed differences become more

280 pronounced as the children grow older, thereby identifying possible mechanisms by which the

281 oral microbiome composition mediates disease. In future analyses, DXA assessment could also

282 be used to explore associations between saliva microbiome and bone remodeling/mass. There

283 is, therefore, need for additional large molecular epidemiologic studies to identify taxonomic and

284 functional links underlying these associations that could be candidates for intervention. 
286 New Hampshire Birth Cohort Study (NHBCS)

287 The NHBCS is an ongoing prospective cohort study of over 2,250 mother and child dyads from

288 New Hampshire and Vermont, USA. The study was originally designed to assess the long-term

289 effect of arsenic exposure from private well water on children born to pregnant women enrolled

290 at approximately 24 and 28 weeks of gestation [28]. Demographic and anthropometric data

291 were collected via interviews administered prenatally, as well as at multiple timepoints

292 postpartum, and via medical record review. Participants provided written informed consent and

293 all study procedures were approved by the Institutional Review Board at Dartmouth College. A

294 subset of children with body composition measurements and saliva shotgun sequencing

295 samples processed when the child was 3 or 4 years old were included in this study.

\section{Childhood growth measurements}

298 Multiple time points and variables were used to describe child growth. In cases when body mass

299 measurements and saliva samples were collected at the same time (within 30 days of each

300 other but generally on the same day), this study design can be considered cross-sectional. For

301 a subset of participants, early life growth measurements (< approximately 2 years of age) were

302 also available and utilized for prospective analyses. Only children that had anthropometric

303 measurements and DXA screening conducted at 3-4 years of age were included in the main

304 analysis assessing associations between body composition and the saliva microbiome cross-

305 sectionally (Supplementary Methods).

306 Body mass measurements at 3-4 years: The anthropometric data were assessed and

307 abstracted from medical records by trained professionals. Data consisted of the following: height

308 (in centimeters) and weight (in kilograms) taken in the clinic during well-child visits when the

309 child was 3 or 4 years of age (1095.75 days $<$ age $<1826.25$ days). BMI was analyzed in $\mathrm{kg} / \mathrm{m}^{2}$

310 and age- and sex-standardized using the CDC's child growth charts derived from the package 
311 childsds [52] in R. Although we considered CDC's referenced growth charts to be a better fit for

312 our cohort of US children, we also computed adjusted BMI z-scores using the World Health

313 Organization's reference charts and found the two methods to be highly correlated (Pearson's

314 correlation $=99 \%$ ). At ages 3 and 4 , children further underwent a full-body DXA scan to

315 estimate body composition using a Horizon-A Advanced Fan-Beam DXA system (Hologic, Inc;

316 Marlborough, MA, USA) following the protocol from the National Institutes of Health PhenX

317 Toolkit [53].

318 Both BMI and DXA measurements were considered to assess child body mass.

319 Although BMI and fat mass both assess child growth, there were a variety of reasons why we

320 explored both in this analysis. We chose to look at BMI because it is a standardized variable

321 that will enable other studies and research groups to compare results with our own. However,

322 as BMI has sensitivity and specificity limitations and DXA has been used as the criterion

323 measure in assessing fat mass in pediatric populations [54,55], we felt it was also important to

324 consider in this study. Thus, we hypothesize that we may be able to identify some trends across

325 both measurements of child growth but may also find varying associations because BMI reflects

326 both fat and fat-free mass. In summary, using both of these measurements of body mass

327 provides advantages to both internal and external validity.

328 Rapid weight gain from birth to age 2 years: Assessment of RWG was conducted to assess a

329 prospective association between rapid child growth early in life and on saliva microbiome

330 composition. RWG has been associated with increased weight and obesity both later in

331 childhood and into adulthood [23, 24]. This score is indicative of the difference between

332 percentile bands on standardized growth charts with 0.67 being the value needed to pass

333 through a centile line [56]. Using delivery and pediatric medical records, we extracted

334 birthweight and a 2-year (+/ 6 months) weight measurement. Children with a gestational age

335 below 37 weeks' gestation were not included in this analysis to reduce potential confounding

336 from gestational age at birth. Weight-for-age z-scores were calculated using the World Health 
337 Organization child growth charts (recommended for clinical use under age 2) and standardized 338 by sex using the childsds [52] in $\mathrm{R}$.

339 Growth chart: In addition to the dichotomous measurement of weight-for-age z-score we

340 modeled growth trajectories of children from birth to 2 years of age using weight-for-

341 length/height ratios. Weight-for-length (or weight-for-height) is recommended as opposed to

342 body mass index in children under 2 years of age [57]. For this analysis, we included children

343 with saliva microbiome samples at 3 or 4 years of age and at least 2 measurements for length

344 and weight before 2 years of age (on or before day 730). Duplicated measurements of weight

345 and height per child were removed and the mean value for the weight-for-length ratio was used

346 if multiple measurements were taken on the same day. Using similar methods to [37], we used

347 the fdapace: Functional Data Analysis and Empirical Dynamics package [58] in R to create

348 growth trajectories for children. This tool enabled us to build growth curves based on the

349 average value across all children. We used the default settings for the FPCA (functional PCA)

350 command.

351

352 Characterization of the salivary microbiome

353 Saliva microbiome samples were collected using flocked nylon swabs (Copan Diagnostics)

354 placed in the child's buccal cavity for 20 seconds to absorb saliva and placed sponge-down into

355 free conical tubes. Sample collection occurred during the 3-to-4-year study visit that included

356 anthropometric assessment and DXA screening. DNA extractions were performed using the

357 ZymoBiomics Micro-prep kit (Zymo Research). Briefly, tubes containing nylon flocked swab

358 heads were thawed and transferred to ZR BashingBead Lysis Tubes ( 0.1 \& $0.5 \mathrm{~mm}$ beads)

359 containing $800 u$ Lysis Buffer and $25 \mathrm{ul}$ Proteinase $\mathrm{K}(20 \mathrm{mg} / \mathrm{ml})$. Lysis tubes were placed in

360 racks in a pre-warmed rotating oven and incubated for 30 minutes $55^{\circ} \mathrm{C}$ and $30 \mathrm{rpm}$. Bead

361 beating of Lysis tubes was performed in two rounds of 11 minutes each using a Disruptor Genie

362 (Scientific Industries, Inc.). After lysis, tubes were centrifuged at 10000xg for 30 seconds in a 
363 microcentrifuge to pellet beads. About 400ul of lysate was transferred to Zymo-Spin ${ }^{\mathrm{TM}}$ III-F Filter

364 columns and centrifuged at $10000 \times \mathrm{xg}$ for 30 seconds and collected in a $2 \mathrm{ml}$ collection tube.

$3651.2 \mathrm{ml}$ of Binding Buffer with $0.5 \%$ beta-mercaptoethanol was mixed with the collected lysate and

366 the mixture was centrifuged through Zymo-Spin ${ }^{\mathrm{TM}}$ IC Columns for $10000 \mathrm{xg}$ for 60 seconds.

367 Columns were washed with kit provided wash and DNA was eluted in 2 pooled elutions, using

368 each time 19 ul of Elution buffer pre-warmed at $60^{\circ} \mathrm{C}$. DNA was quantified using Qubit HS

369 dsDNA kit (Invitrogen) and 2ul of sample. A yield threshold of 1ng/ul DNA was required to refer

370 for shotgun sequencing. Above this threshold average DNA yield was $8.6 \pm 9.8 \mathrm{ng} / \mathrm{ul}$ and

371 ranged from 1 to $57.7 \mathrm{ng} / \mathrm{ul}$. DNA extractions were performed in batches of 12 samples

372 including one external saliva positive control swab and one negative control swab. Average

373 DNA yields of batch positive controls was $15.2 \mathrm{ng} / \mathrm{ul}$ and $\mathrm{CV}$ of $19 \%$. Negative control yields

374 were too low to quantify at 2ul. Extracted DNA was amplified from all samples were prepared for

375 sequencing on the NextSeq platform (shotgun metagenomics) using $150 \mathrm{nt}$ paired end reads at

376 the Marine Biological Laboratory (MBL) in Woods Hole, MA using established methods and as

377 previously published [28, 59-61].

378 All samples were processed as single reads at the MBL and were subsequently

379 processed. First, they underwent quality control to remove contaminants with KneadData v0.7.4.

380 Only saliva samples that had one million reads after KneadData processing were kept in the

381 analysis. Shotgun sequencing samples were functionally profiled using HUMAnN3 version

382 3.0.0.alpha.3 [62] after being taxonomically profiled using MetaPhIAn3 [63]. MetaPhIAn3 and

383 HUMAnN3 jobs were run on Dartmouth's supercomputer and high-performance computing

384 Linux cluster respectively. HUMAnN3 uses a tiered search approach to first map reads from

385 samples to taxa using marker genes (MetaPhIAn3). Then it creates species-specific

386 pangenomes to provide functional annotations [62]. Only reads from bacteria were considered

387 for this analysis which made up the vast majority (> 99\%) of all samples. 


\section{Data analysis}

390 Covariates: Covariates were selected based on an a priori literature review. In order to choose

391 which covariates were confounders and needed to be adjusted for in the models, we plotted

392 them on a directed acyclic graph (DAG) (Figure S5). Although our measurement of body mass

393 and saliva microbiome were cross-sectional in our main analysis, we hypothesize a direction of

394 association with body mass as the exposure and the saliva microbiome as the outcome. This

395 was directly examined in our prospective analyses by assessing child growth between 0 and 2

396 years of age. Based on the DAG, the potential confounders (i.e., related to our exposure and

397 outcome directly or hypothesized to be based on previously identified indirect associations in

398 separate studies) to adjust for in our analyses were age (measured by age in days of the saliva

399 sample), sex (male or female)[37, 60], delivery mode (vaginal or cesarean delivery)[3],

400 gestational age (age in weeks)[27, 61] and diet (age when child started to eat solid foods in

401 months) [37]. Although previous studies have found associations between maternal BMI or

402 weight gain and children's BMI [64], literature identifying associations between maternal BMI

403 and the child's saliva microbiome have not found associations [37, 65]. However, due to

404 previously identified associations between maternal BMI and the child's gut microbiota [66], we

405 decided to include maternal BMI measured by self-reported pre-pregnancy weight and

406 measured height (kg per meters squared) as a covariate.

408 Descriptive and statistical analyses: All analyses were completed in R 3.6.0 (http://www.R-

409 project.org). Saliva microbiota sequence read counts were normalized per sample, yielding a

410 compositional relative abundance data set for downstream analyses. We were interested in

411 taxonomic composition at the phylum, genus, and species-level. Each read was classified using

412 the CHOCOPhIAn database within MetaPhIAn3, and, for each sample, ecological diversity

413 measurements ( $\alpha$-diversity - a measure of richness and evenness within a single sample, and

$414 \beta$-diversity - a measure of differences between samples) were calculated at the species-level. 
To evaluate the relationship between alpha diversity and childhood growth measures,

416 the phyloseq package [67] was used to compute Shannon diversity. In statistical models, the

417 Shannon alpha diversity was the outcome and was regressed against the relevant exposures

418 using linear regression models. In these models, TFM was transformed into kilograms. Between

419 sample or beta diversity was also assessed using the phyloseq package. Bray-Curtis was used

420 to assess dissimilarity between samples and plotted as a principal coordinate analysis (PCoA)

421 plot to visualize variation. Samples were colored by variables of interest. For overweight status,

422 centroids were plotted with betadisper function within the vegan package (ellipses based on 1

423 standard deviation). Variation between samples was quantified using the adonis2 function from

424 the vegan package [68] in PERMANOVA analyses. Lastly, Microbiome Multivariable

425 Associations with Linear Models (MaAsLin2) [69] was used to quantify differences between the

426 relative abundance of taxa by body mass variables adjusted for covariates. MaAsLin2 is

427 specifically designed for 'omic analyses and uses robust statistical procedures to assess

428 exposures of interest while controlling for other variables. Deviation from default parameters for

429 taxonomic analysis included normalization though the centered-log ratio (CLR) approach and no

430 additional transformation. Interaction variables consider the joint effect of the child growth

431 variable among children in one group (i.e., the joint interaction of female sex and BMI).

432 MaAsLin2 for pathways and KEGG gene family analysis used default parameters with the

433 additional minimum abundance filtering of 0.0001 . Due to the hypothesis-generating and

434 exploratory nature of this study, effect size, $p$-value, and q-value thresholds were used to

435 determine taxa, genes, and pathways of interest, but thresholds varied by analysis. Thresholds

436 for inclusion in figures are noted in figure legends.

439 Funding: This work was funded in part by the US National Institute Health under award 440 numbers UH3OD023275, NIAID T32AI007519, NIEHS P01ES022832, NIEHS P20ES018175, 
442 under award numbers RD83459901 and RD83544201.

443 Data availability: The saliva whole metagenomic shotgun sequencing samples are available

444 through the National Center for Biotechnology (NCBI) Sequence Read Archive:

445 https://www.ncbi.nlm.nih.gov/sra (Accession number: PRJNA296814).

446 Code availability: The authors will share Rmarkdown scripts upon request.

\section{References}

449 1. Fryar CD, C.M., Afful J, Prevalence of overweight, obesity, and severe obesity among $450 \quad$ children and adolescents aged 2-19 years: United States, 1963-1965 through 2017451 2018. 2020.

452 2. Khan, M.J., et al., Role of Gut Microbiota in the Aetiology of Obesity: Proposed

453 Mechanisms and Review of the Literature. J Obes, 2016. 2016: p. 7353642.

3. DiBaise, J.K., et al., Gut microbiota and its possible relationship with obesity. Mayo Clin Proc, 2008. 83(4): p. 460-9.

4. Ley, R.E., et al., Obesity alters gut microbial ecology. Proc Natl Acad Sci U S A, 2005. 102(31): p. 11070-5.

5. Turnbaugh, P.J., et al., An obesity-associated gut microbiome with increased capacity for energy harvest. Nature, 2006. 444(7122): p. 1027-31.

6. Zhao, L., The gut microbiota and obesity: from correlation to causality. Nat Rev Microbiol, 2013. 11(9): p. 639-47.

7. Cho, K.Y., Lifestyle modifications result in alterations in the gut microbiota in obese children. BMC Microbiol, 2021. 21(1): p. 10.

464 8. Sfasciotti, G.L., et al., Childhood overweight-obesity and periodontal diseases: is there a real correlation? Ann Stomatol (Roma), 2016. 7(3): p. 65-72.

9. Han, Y.W. and X. Wang, Mobile microbiome: oral bacteria in extra-oral infections and inflammation. J Dent Res, 2013. 92(6): p. 485-91.

10. Wade, W.G., The oral microbiome in health and disease. Pharmacol Res, 2013. 69(1): p. 137-43.

11. Nikitakis, N.G., et al., The autoimmunity-oral microbiome connection. Oral Dis, 2016.

12. Wu, Y., et al., Characterization of the salivary microbiome in people with obesity. PeerJ, 2018. 6: p. e4458.

13. Mervish, N.A., et al., Associations of the Oral Microbiota with Obesity and Menarche in Inner City Girls. J Child Obes, 2019. 4(1).

14. Wang, R.R., et al., Association of the oral microbiome with the progression of impaired fasting glucose in a Chinese elderly population. J Oral Microbiol, 2019. 11(1): p. 1605789. 
15. Araujo, D.S., et al., Salivary Microbiological and Gingival Health Status Evaluation of Adolescents With Overweight and Obesity: A Cluster Analysis. Front Pediatr, 2020. 8: p. 429.

16. Ding, T. and P.D. Schloss, Dynamics and associations of microbial community types across the human body. Nature, 2014. 509(7500): p. 357-60.

17. Oba, P.M., et al., Diet Influences the Oral Microbiota of Infants during the First Six Months of Life. Nutrients, 2020. 12(11).

18. Eshriqui, I., et al., Breastfeeding may have a long-term effect on oral microbiota: results from the Fin-HIT cohort. Int Breastfeed J, 2020. 15(1): p. 42.

19. Derrien, M., A.S. Alvarez, and W.M. de Vos, The Gut Microbiota in the First Decade of Life. Trends Microbiol, 2019. 27(12): p. 997-1010.

20. Moore, R.E. and S.D. Townsend, Temporal development of the infant gut microbiome. Open Biol, 2019. 9(9): p. 190128.

21. Stewart, C.J., et al., Temporal development of the gut microbiome in early childhood from the TEDDY study. Nature, 2018. 562(7728): p. 583-588.

22. Geserick, M., et al., Acceleration of BMI in Early Childhood and Risk of Sustained Obesity. N Engl J Med, 2018. 379(14): p. 1303-1312.

23. Rotevatn, T.A., et al., Infancy weight gain, parental socioeconomic position, and childhood overweight and obesity: a Danish register-based cohort study. BMC Public Health, 2019. 19(1): p. 1209.

24. Monteiro, P.O. and C.G. Victora, Rapid growth in infancy and childhood and obesity in later life--a systematic review. Obes Rev, 2005. 6(2): p. 143-54.

25. Bell, K.A., et al., Validity of Body Mass Index as a Measure of Adiposity in Infancy. J Pediatr, 2018. 196: p. 168-174 e1.

26. Ley, R.E., et al., Microbial ecology: human gut microbes associated with obesity. Nature, 2006. 444(7122): p. 1022-3.

27. Coker, M.O., et al., Infant Feeding Alters the Longitudinal Impact of Birth Mode on the Development of the Gut Microbiota in the First Year of Life. Front Microbiol, 2021. 12: p. 642197.

28. Madan, J.C., et al., Association of Cesarean Delivery and Formula Supplementation With the Intestinal Microbiome of 6-Week-Old Infants. JAMA Pediatr, 2016. 170(3): p. 212-9.

29. Bervoets, L., et al., Differences in gut microbiota composition between obese and lean children: a cross-sectional study. Gut Pathogens, 2013. 5.

30. Rinninella, E., et al., What is the Healthy Gut Microbiota Composition? A Changing Ecosystem across Age, Environment, Diet, and Diseases. Microorganisms, 2019. 7(1).

31. Wang, X., et al., Bioinspired oral delivery of gut microbiota by self-coating with biofilms. Sci Adv, 2020. 6(26): p. eabb1952.

32. Janem, W.F., et al., Salivary inflammatory markers and microbiome in normoglycemic lean and obese children compared to obese children with type 2 diabetes. PLoS One, 2017. 12(3): p. e0172647.

33. Besnard, P., et al., Obese Subjects With Specific Gustatory Papillae Microbiota and Salivary Cues Display an Impairment to Sense Lipids. Sci Rep, 2018. 8(1): p. 6742.

34. Zeigler, C.C., et al., Microbiota in the Oral Subgingival Biofilm Is Associated With Obesity in Adolescence. Obesity, 2012. 20(1): p. 157-164. 
35. Yang, Y., et al., Oral microbiome and obesity in a large study of low-income and AfricanAmerican populations. J Oral Microbiol, 2019. 11(1): p. 1650597.

36. Vonaesch, P., et al., Stunted childhood growth is associated with decompartmentalization of the gastrointestinal tract and overgrowth of oropharyngeal taxa. Proceedings of the National Academy of Sciences, 2018. 115(36): p. E8489-E8498.

37. Craig, S.J.C., et al., Child Weight Gain Trajectories Linked To Oral Microbiota Composition. Sci Rep, 2018. 8(1): p. 14030.

38. Nearing, J.T., et al., Assessing the Variation within the Oral Microbiome of Healthy Adults. Msphere, 2020. 5(5).

39. Raju, S.C., et al., Gender-Specific Associations Between Saliva Microbiota and Body Size. Frontiers in Microbiology, 2019. 10.

40. Mameli, C., et al., Taste perception and oral microbiota are associated with obesity in children and adolescents. PLoS One, 2019. 14(9): p. e0221656.

41. Goodson, J.M., et al., Is Obesity an Oral Bacterial Disease? Journal of Dental Research, 2009. 88(6): p. 519-523.

42. Herrera, D., et al., Antimicrobial therapy in periodontitis: the use of systemic antimicrobials against the subgingival biofilm. J Clin Periodontol, 2008. 35(8 Suppl): p. 45-66.

43. Mbakwa, C.A., et al., Gut Microbiota and Body Weight in School-Aged Children: The KOALA Birth Cohort Study. Obesity (Silver Spring), 2018. 26(11): p. 1767-1776.

44. Larsen, J.M., The immune response to Prevotella bacteria in chronic inflammatory disease. Immunology, 2017. 151(4): p. 363-374.

45. Keller, A., et al., Association Between Periodontal Disease and Overweight and Obesity: A Systematic Review. Journal of Periodontology, 2015. 86(6): p. 766-776.

46. Slocum, C., C. Kramer, and C.A. Genco, Immune dysregulation mediated by the oral microbiome: potential link to chronic inflammation and atherosclerosis. J Intern Med, 2016. 280(1): p. 114-28.

47. Shin, Y.L., The Timing of Rapid Infant Weight Gain in Relation to Childhood Obesity. J Obes Metab Syndr, 2019. 28(4): p. 213-215.

48. Costa N, S.P., Ferreira A, et al. , Maternal Pre-Pregnancy Body Mass Index and Gestational Weight Gain Are Associated with Differences in Infant Gut Microbiota: Results from Brazilian Prospective Birth Cohort. Curr Dev Nutr., 2020. 4.

49. Francis, L.A., S.M. Hofer, and L.L. Birch, Predictors of maternal child-feeding style: maternal and child characteristics. Appetite, 2001. 37(3): p. 231-43.

50. van den Bogert, B., et al., Immunomodulatory properties of Streptococcus and Veillonella isolates from the human small intestine microbiota. PLoS One, 2014. 9(12): p. e114277.

51. Egland, P.G., R.J. Palmer, Jr., and P.E. Kolenbrander, Interspecies communication in Streptococcus gordonii-Veillonella atypica biofilms: signaling in flow conditions requires juxtaposition. Proc Natl Acad Sci U S A, 2004. 101(48): p. 16917-22.

52. Vogel M, childsds: Data and Methods Around Reference Values in Pediatrics. 2020, R. 53. Hamilton, C.M., et al., The PhenX Toolkit: Get the Most From Your Measures. American Journal of Epidemiology, 2011. 174(3): p. 253-260. 
54. Eisenmann, J.C., K.A. Heelan, and G.J. Welk, Assessing body composition among 3-to 81633-1640.

55. Orsso, C.E., et al., Assessment of body composition in pediatric overweight and obesity: A 21(8): p. e13041.

56. Zheng, W., et al., Longitudinal changes in body mass index of children affected by the

Great East Japan Earthquake. Int J Obes (Lond), 2017. 41(4): p. 606-612.

57. Centers for Disease Control and Prevention, Growth Chart Training : Using the WHO Growth Charts. 2015.

58. Carroll, C., et al. Functional Data Analysis and Empirical Dynamics [R package fdapace version 0.5.5]. 2020.

59. Coker, M.O., et al., Specific class of intrapartum antibiotics relates to maturation of the infant gut microbiota: a prospective cohort study. BJOG, 2019.

60. Hoen, A.G., et al., Sex-specific associations of infants' gut microbiome with arsenic exposure in a US population. Sci Rep, 2018. 8(1): p. 12627.

61. Lebeaux, R.M., et al., The infant gut resistome is associated with E. coli and early-life exposures. BMC Microbiol, 2021. 21(1): p. 201.

62. Franzosa, E.A., et al., Species-level functional profiling of metagenomes and metatranscriptomes. Nat Methods, 2018. 15(11): p. 962-968.

63. Beghini, F., et al., Integrating taxonomic, functional, and strain-level profiling of diverse microbial communities with bioBakery 3. bioRxiv, 2020: p. 2020.11.19.388223.

64. Heslehurst, N., et al., The association between maternal body mass index and child obesity: A systematic review and meta-analysis. PLoS Med, 2019. 16(6): p. e1002817.

65. Gomez-Arango, L.F., et al., Contributions of the maternal oral and gut microbiome to placental microbial colonization in overweight and obese pregnant women. Sci Rep, 2017. 7(1): p. 2860.

66. Galley, J.D., et al., Maternal obesity is associated with alterations in the gut microbiome in toddlers. PLoS One, 2014. 9(11): p. e113026.

67. McMurdie, P.J. and S. Holmes, phyloseq: an $R$ package for reproducible interactive analysis and graphics of microbiome census data. PLoS One, 2013. 8(4): p. e61217.

68. Dixon, P., VEGAN, a package of $R$ functions for community ecology. Journal of Vegetation Science, 2003. 14(6): p. 927-930.

69. Mallick, H., et al., Multivariable Association Discovery in Population-scale Meta-omics Studies. bioRxiv, 2021: p. 2021.01.20.427420. 
A

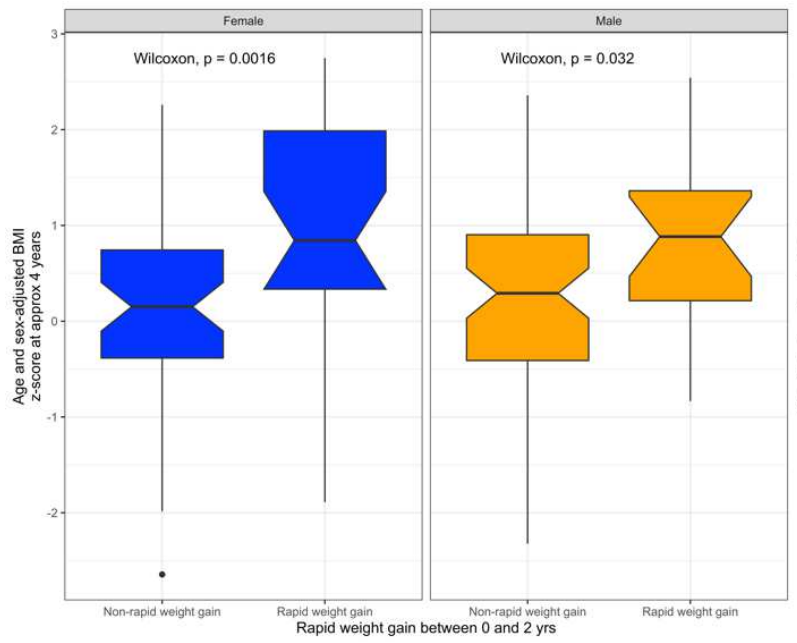

B

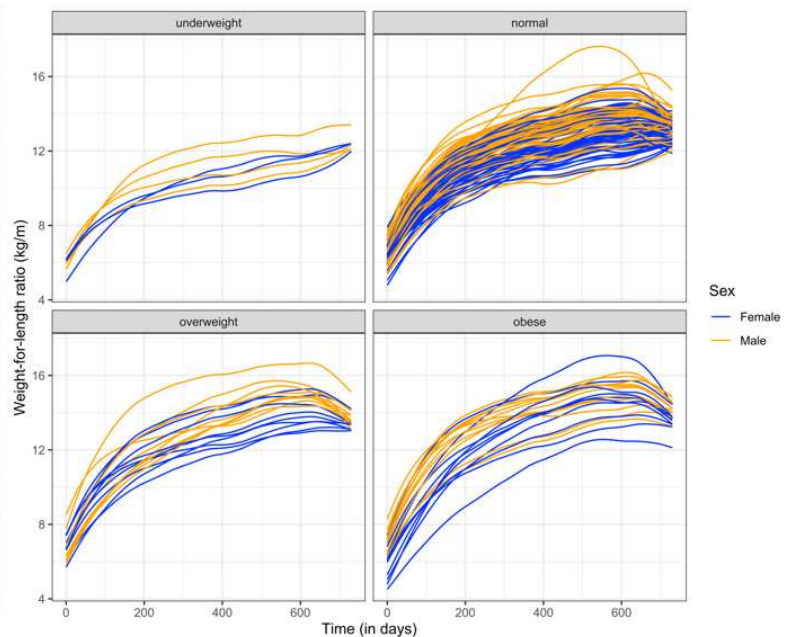

Figure 1: Associations between body mass index (BMI) measured at 3 or 4 years of age versus child growth metrics measured between 0 and 2 years of age. A) Age and sex adjusted BMI z-score versus rapid weight gain stratified by sex for 157 children. Rapid weight gain was defined as a $>0.67$ increase in weight-for-age $z$-score between 0 and 2 years of age with 0.67 indicative of the difference between percentile bands measured on a standardized growth chart. Wilcoxon p-value indicates difference in BMI z-score by rapid weight gain group for females and males. B) Growth charts using the weight-for-length (weight-for-height) ratio plotted for 195 children between the ages of 0 and 2 stratified by BMI percentile groupings at 3 or 4 years of age. Growth indices are colored by the sex of the child. 

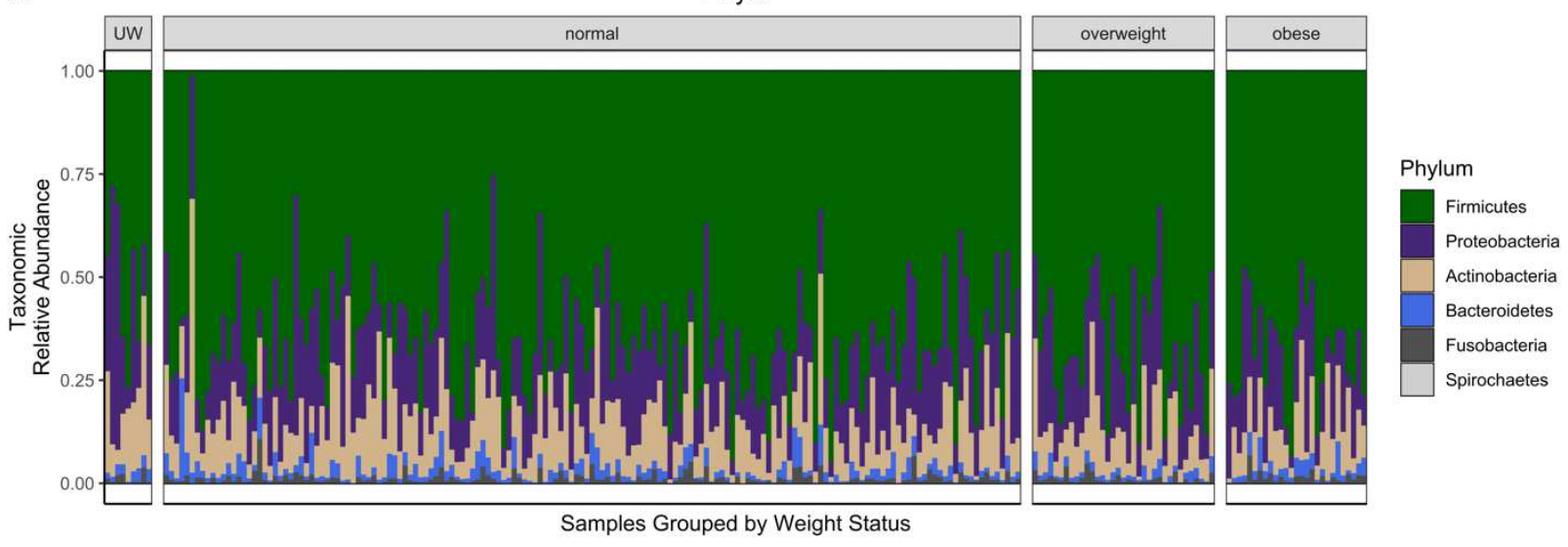

B
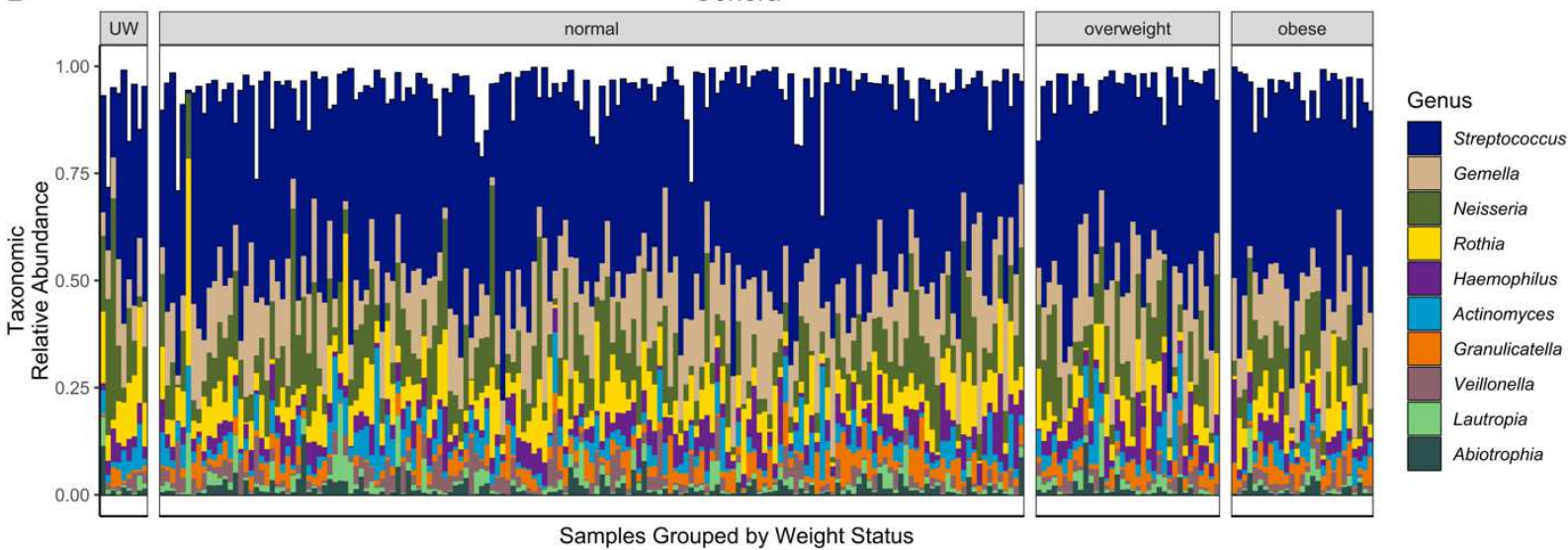

C Species
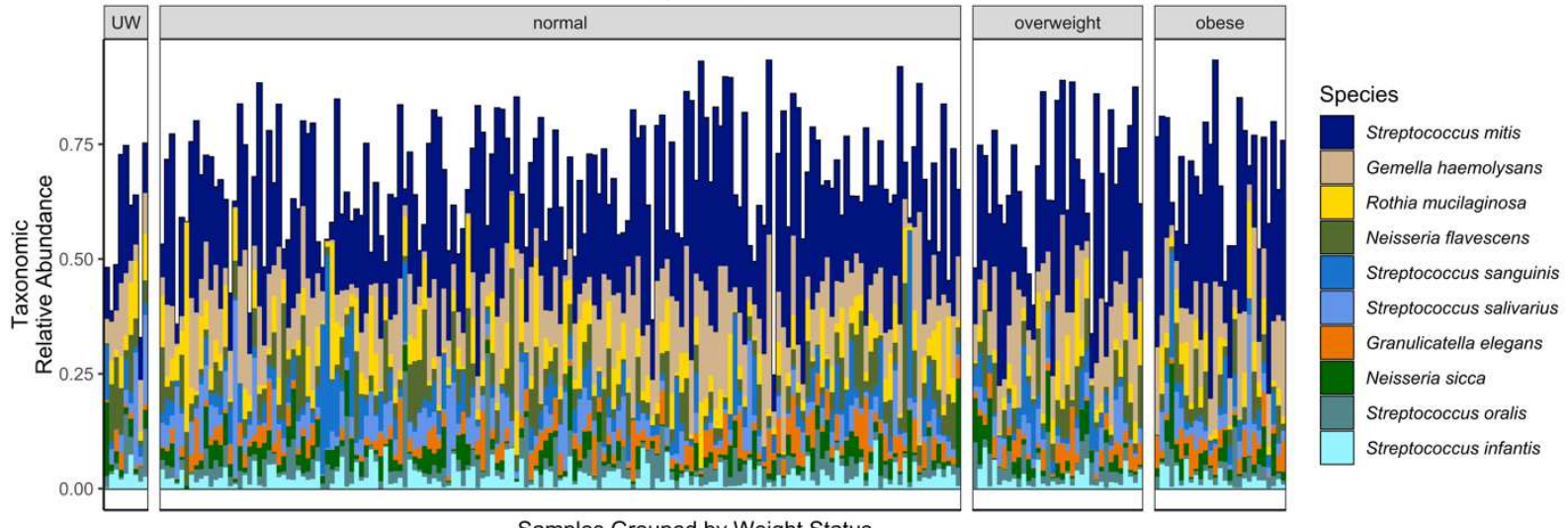

Figure 2: Saliva microbiome composition among 236 children grouped by body mass index percentile groupings. A) Phylum-specific composition by mean relative abundance. B) The composition of the top 10 genera based on the highest mean relative abundance across all samples. C) The composition of the top 10 species based on the highest mean relative abundance across all samples. Color ranges (i.e., spectrum of blue for Streptococcus) for species are used to delineate species from the same genus. UW = underweight 
A

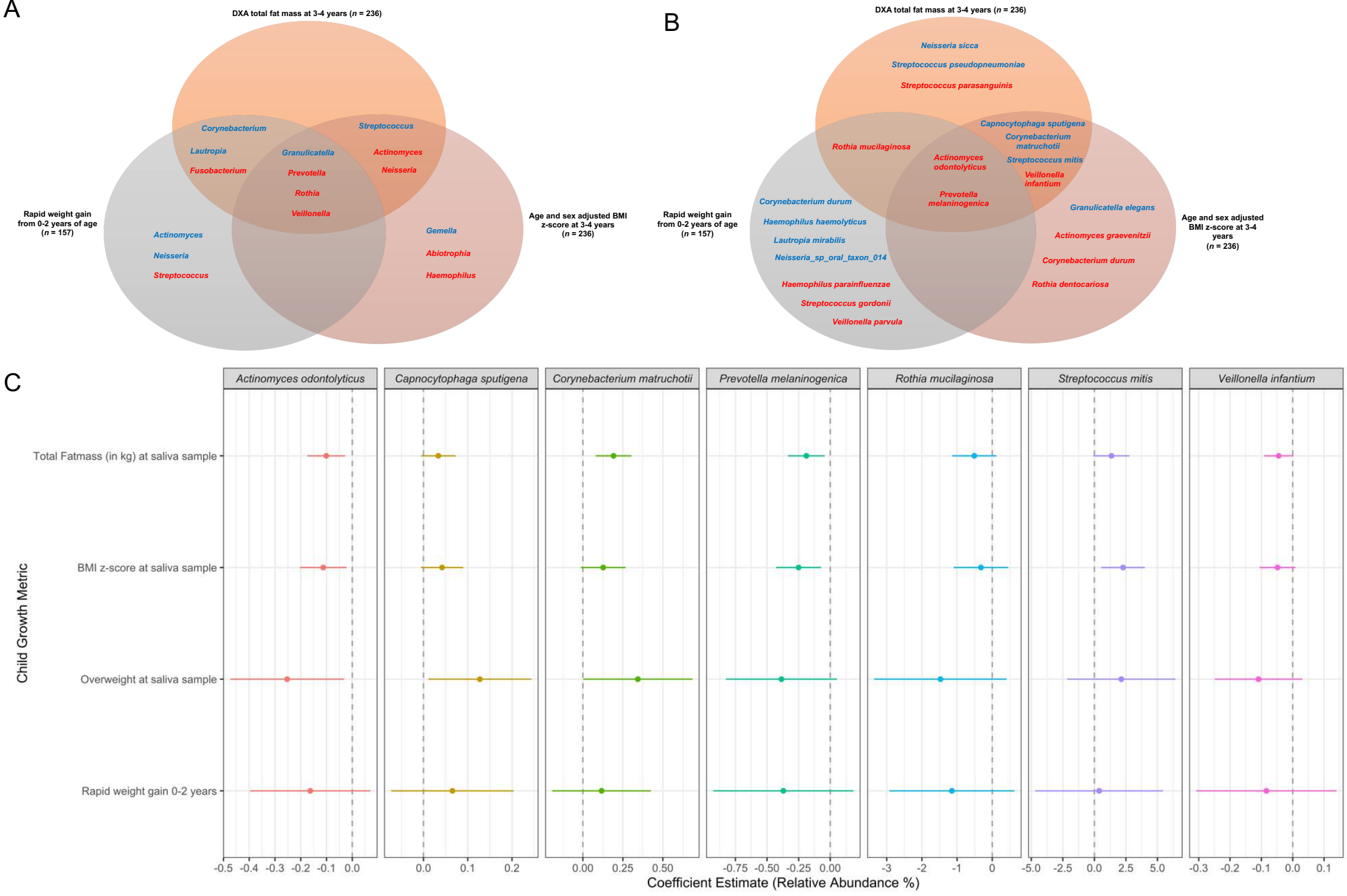


Figure 3: Comparison of associations between growth metrics and microbes. A) Venn diagram depicting the top 10 genera by lowest p-value produced from adjusted MaAsLin2 regressions. B) Venn diagram depicting the top 10 species by lowest $p$-value. For both $A$ and $B$, blue and red are indicative of positive and negative coefficients respectively. $C$ ) Dot and whisker plots to represent the relative abundance change attributable to the child growth metric. Each row represents the coefficient estimate from a different linear regression model. These adjusted regression models included the exposure (growth variable) and the following covariates: delivery mode (vaginal or cesarean), sex (male or female), sample age in days, maternal BMI, gestational age in weeks, and solid foods start age in months. The sample size of the adjusted models for the growth metrics measured at the time of saliva sample collection was 202 . The sample size for the rapid weight gain model was 138. Species were selected for univariate linear regression analysis due to their overlap in the species-level Venn diagram. 
A

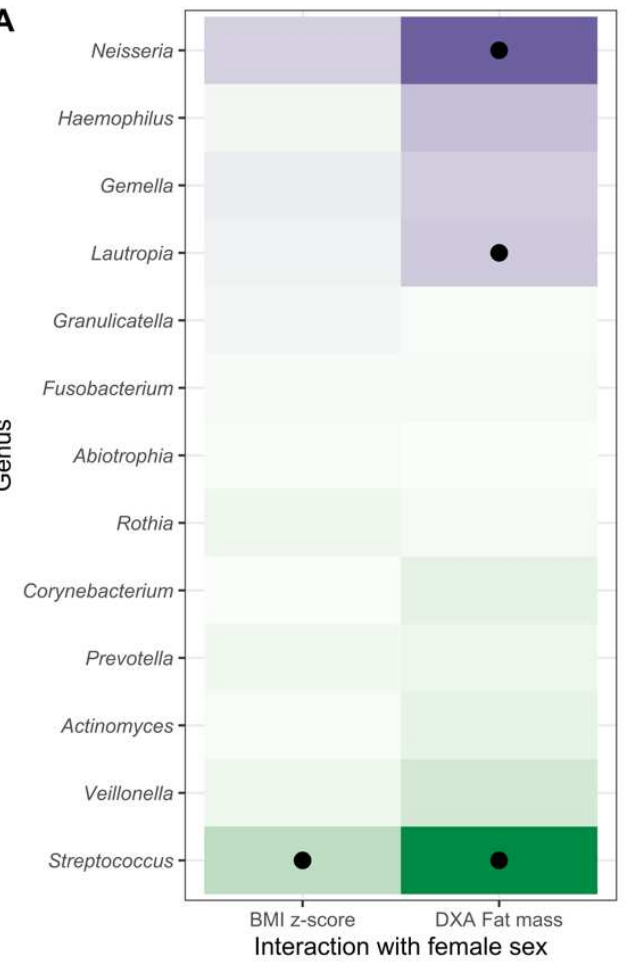

C

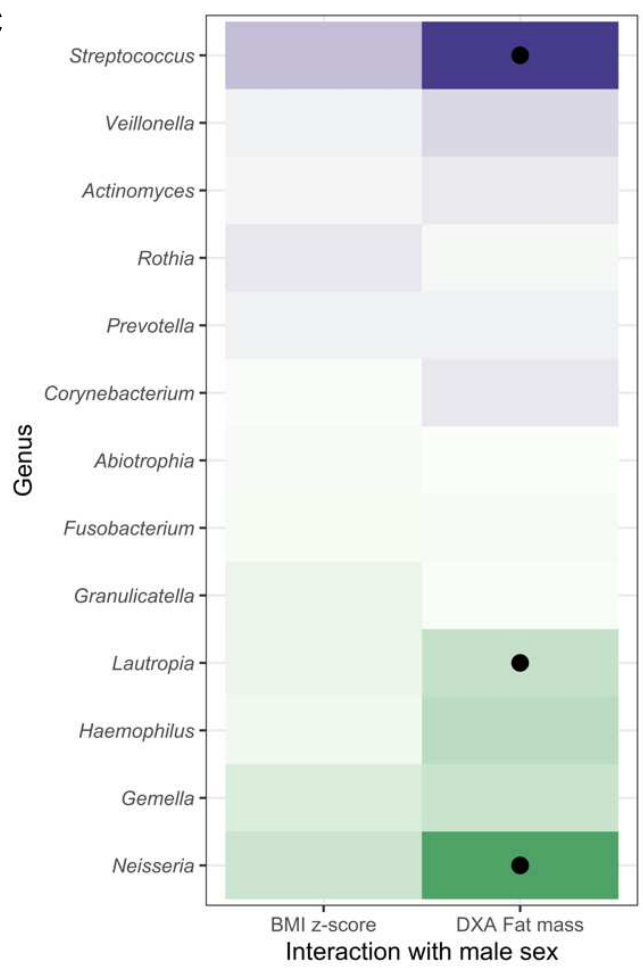

B

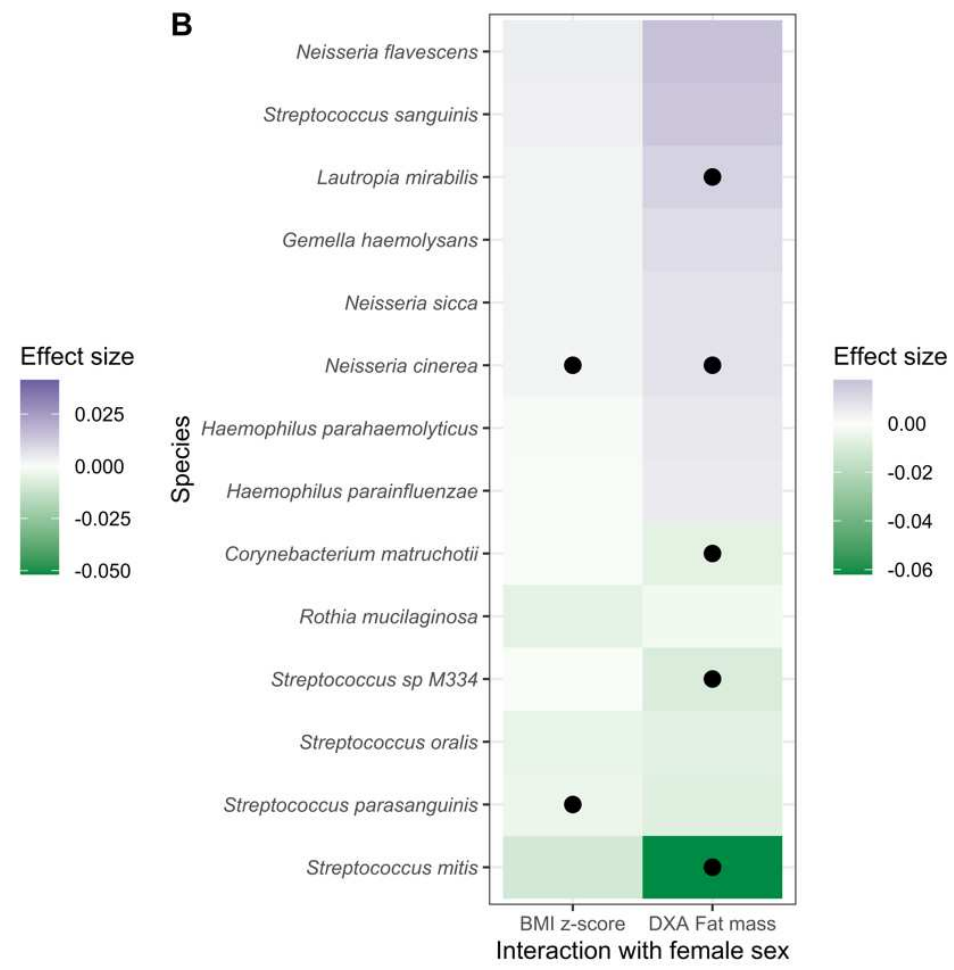

D

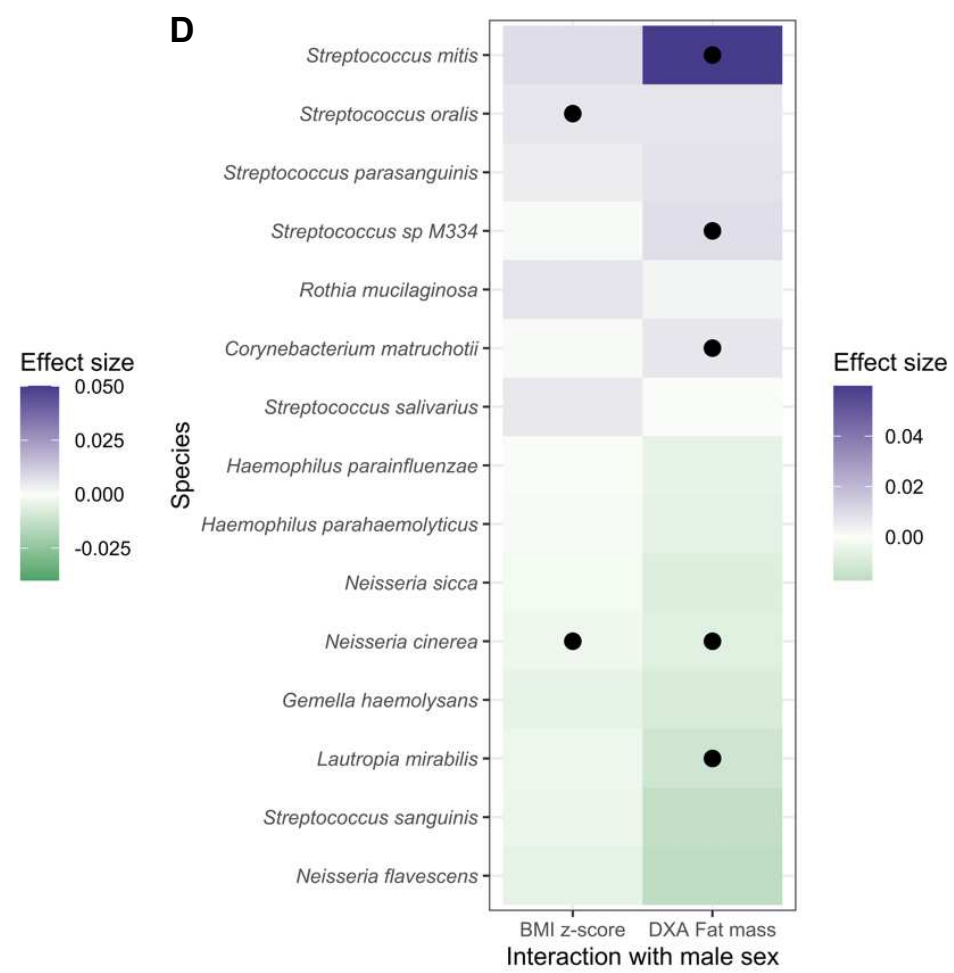

Figure 4: Assessing the joint effects of body mass and sex on saliva microbiota at 3 or 4 years of age. MaAsLin2 models included in addition to the interaction term: the growth 
variable, sex (male or female), delivery mode (vaginal or cesarean), sample age in days, maternal BMl, gestational age in weeks, and solid foods start age in months.

Black circles indicate a $p$-value $<0.1$. A) Coefficient for interaction between female sex and child growth metric (age and sex adjusted BMI z-score or total fat mass in grams using a DXA scan) on the relative abundance of genera. B) Coefficient for the interaction between female sex and child growth metrics on the relative abundance of species. $\mathbf{C}$ and $\mathbf{D}$ use the same data but the models for the interaction term represents joint effects with males instead of females. For species, only associations with an effect size $>0.005$ or $<-0.005$ are included. 

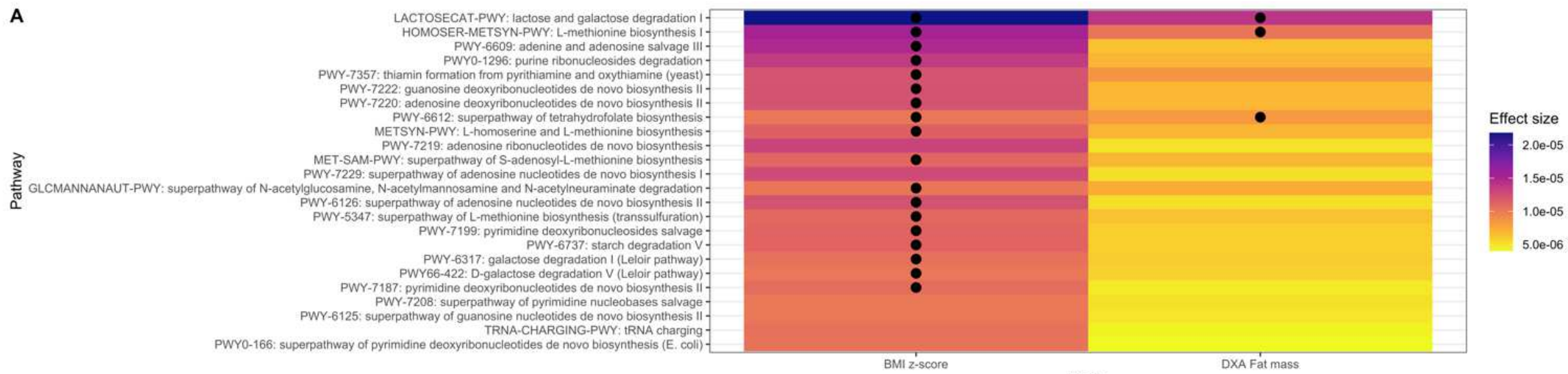

B
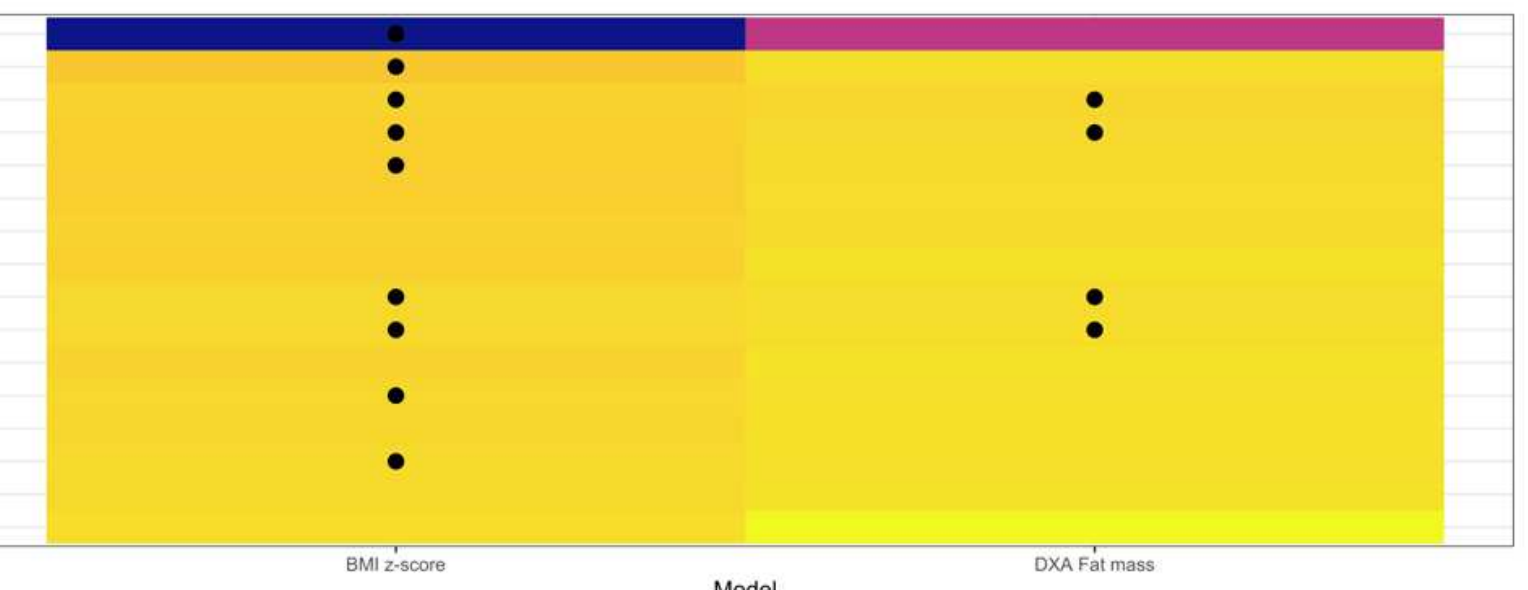

Model

Model

Figure 5: Tile plots showing associations between body mass metrics and saliva microbiome functional profiles. Effect sizes are derived from MaAsLin2 analyses. The models were adjusted for delivery mode (vaginal or cesarean), sex (male or female), sample age in days, maternal BMI, gestational age in weeks, and solid foods start age in months. Black circles represent a p-value $<0.15$. A) Pathway relative abundance by child growth metrics (age and sex-adjusted BMI and DXA measured total fat mass in kilograms). Only associations with pathways with an effect size absolute value greater than 0.00001 in at least one of the two models are included. B) KEGG gene family relative abundance by child growth metrics. Only gene families with an effect size absolute value greater than 0.000005 in at least one of the 2 models are included. 
Table 1. Descriptive overview of children with BMI, DXA, and saliva microbiome samples at 3-4 years of age by BMI percentile-based groups

\begin{tabular}{|c|c|c|c|c|c|}
\hline & Underweight & $\begin{array}{c}\text { Normal weight } \\
5^{\text {th }} \text { to } 85^{\text {th }}\end{array}$ & $\begin{array}{l}\text { Overweight } \\
85^{\text {th }} \text { to } 95^{\text {th }}\end{array}$ & $\begin{array}{l}\text { Obese } \\
\geq 95^{\text {th }}\end{array}$ & Overall \\
\hline BMI percentile & $<5^{\text {th }}$ percentile & percentile & percentile & percentile & \\
\hline $\begin{array}{c}\text { Number of children by } \\
\text { group }\end{array}$ & $9(3.8 \%)$ & $165(69.9 \%)$ & $35(14.8 \%)$ & $27(11.4 \%)$ & $236(100 \%)$ \\
\hline \multicolumn{6}{|l|}{$\begin{array}{c}\text { Sample age of saliva } \\
\text { sample (days) }\end{array}$} \\
\hline Mean (SD) & $1420(87.4)$ & $1410(82.3)$ & $1400(64.1)$ & $1430(98.2)$ & $1410(81.9)$ \\
\hline Median [Min, Max] & $\begin{array}{c}1390[1320 \\
1600]\end{array}$ & $\begin{array}{c}1390[1160 \\
1750]\end{array}$ & $\begin{array}{c}1400[1310 \\
1550]\end{array}$ & $\begin{array}{c}1410[1310, \\
1680]\end{array}$ & $\begin{array}{c}1390[1160 \\
1750]\end{array}$ \\
\hline \multicolumn{6}{|l|}{ Sex } \\
\hline Male & $6(66.7 \%)$ & $90(54.5 \%)$ & $16(45.7 \%)$ & $19(70.4 \%)$ & $131(55.5 \%)$ \\
\hline Female & $3(33.3 \%)$ & $75(45.5 \%)$ & $19(54.3 \%)$ & $8(29.6 \%)$ & $105(44.5 \%)$ \\
\hline \multicolumn{6}{|l|}{ Maternal BMI $\left(\mathrm{kg} / \mathrm{m}^{2}\right)$} \\
\hline Mean (SD) & $21.9(1.92)$ & $25.4(5.02)$ & $26.5(5.35)$ & $29.0(6.46)$ & $25.8(5.33)$ \\
\hline Median [Min, Max] & $22.4[18.3,24.4]$ & $24.1[17.5,45.7]$ & $\begin{array}{c}25.4[18.7 \\
41.5]\end{array}$ & $\begin{array}{l}27.0[21.5 \\
45.2]\end{array}$ & $\begin{array}{c}24.4[17.5 \\
45.7]\end{array}$ \\
\hline Missing & $0(0 \%)$ & $4(2.4 \%)$ & $2(5.7 \%)$ & $0(0 \%)$ & $6(2.5 \%)$ \\
\hline \multicolumn{6}{|l|}{ Delivery method } \\
\hline Vaginal & $8(88.9 \%)$ & $120(72.7 \%)$ & $23(65.7 \%)$ & $19(70.4 \%)$ & $170(72.0 \%)$ \\
\hline C-section & $1(11.1 \%)$ & $45(27.3 \%)$ & $11(31.4 \%)$ & $8(29.6 \%)$ & $65(27.5 \%)$ \\
\hline Missing & $0(0 \%)$ & $0(0 \%)$ & $1(2.9 \%)$ & $0(0 \%)$ & $1(0.4 \%)$ \\
\hline \multicolumn{6}{|l|}{$\begin{array}{l}\text { Gestational age at birth } \\
\text { (weeks) }\end{array}$} \\
\hline Mean (SD) & $38.5(1.24)$ & $39.0(1.83)$ & $38.9(2.27)$ & $38.9(1.88)$ & $39.0(1.88)$ \\
\hline Median [Min, Max] & $38.6[36.7,40.0]$ & $39.3[31.6,42.0]$ & $\begin{array}{l}39.0[31.0 \\
43.0]\end{array}$ & $\begin{array}{c}39.3[34.3 \\
41.4]\end{array}$ & $\begin{array}{l}39.1[31.0 \\
43.0]\end{array}$ \\
\hline \multicolumn{6}{|l|}{$\begin{array}{l}\text { Solid foods start age } \\
\text { (months) }\end{array}$} \\
\hline Mean (SD) & $5.44(1.59)$ & $5.36(1.28)$ & $5.07(1.33)$ & $4.92(1.22)$ & $5.28(1.30)$ \\
\hline Median [Min, Max] & $6.00[3.00,8.00]$ & $6.00[1.00,10.0]$ & $\begin{array}{l}5.00[3.00 \\
8.00]\end{array}$ & $\begin{array}{l}5.00[3.00 \\
8.00]\end{array}$ & $\begin{array}{c}5.00[1.00 \\
10.0]\end{array}$ \\
\hline Missing & $0(0 \%)$ & $19(11.5 \%)$ & $6(17.1 \%)$ & $5(18.5 \%)$ & $30(12.7 \%)$ \\
\hline \multicolumn{6}{|l|}{$\begin{array}{c}\text { Body Mass Index } \\
\left(\mathrm{kg} / \mathrm{m}^{2}\right)\end{array}$} \\
\hline Mean (SD) & $13.5(0.256)$ & $15.6(0.762)$ & $17.4(0.301)$ & $18.8(0.716)$ & $16.2(1.40)$ \\
\hline Median [Min, Max] & $13.6[13.1,13.9]$ & $15.7[13.7,17.0]$ & $\begin{array}{c}17.3[16.9 \\
18.0] \\
\end{array}$ & $\begin{array}{c}18.8[17.9 \\
21.3] \\
\end{array}$ & $\begin{array}{c}16.0[13.1 \\
21.3]\end{array}$ \\
\hline \multicolumn{6}{|l|}{ Height $(\mathrm{cm})$} \\
\hline Mean (SD) & $100(4.50)$ & $102(4.18)$ & $102(3.42)$ & $104(4.84)$ & $102(4.26)$ \\
\hline Median [Min, Max] & $98.3[92.9,106]$ & $102[91.2,117]$ & $\begin{array}{c}101[95.7 \\
109]\end{array}$ & $\begin{array}{c}104[96.2 \\
115]\end{array}$ & $\begin{array}{c}102[91.2 \\
117]\end{array}$ \\
\hline \multicolumn{6}{|l|}{ Weight (kg) } \\
\hline Mean (SD) & $13.5(1.11)$ & $16.2(1.61)$ & $18.0(1.26)$ & $20.6(2.08)$ & $16.8(2.26)$ \\
\hline Median [Min, Max] & $13.1[12.0,15.1]$ & $16.2[12.9,19.6]$ & $\begin{array}{c}17.9[15.5 \\
21.1] \\
\end{array}$ & $\begin{array}{c}20.4[16.7 \\
25.0]\end{array}$ & $\begin{array}{l}16.7[12.0 \\
25.0] \\
\end{array}$ \\
\hline \multicolumn{6}{|l|}{ Total fat mass $(\mathrm{g})$} \\
\hline Mean (SD) & $3290(644)$ & $4920(898)$ & $6200(989)$ & $7370(1350)$ & $5330(1340)$ \\
\hline Median [Min, Max] & $\begin{array}{c}3340[1970 \\
3990]\end{array}$ & $\begin{array}{c}5000[2550, \\
7430]\end{array}$ & $\begin{array}{c}6010[3980 \\
7940]\end{array}$ & $\begin{array}{c}7330[5050 \\
10500]\end{array}$ & $\begin{array}{c}5200[1970, \\
10500]\end{array}$ \\
\hline \multicolumn{6}{|l|}{ Total lean mass (g) } \\
\hline Mean (SD) & $9860(1100)$ & $10900(1300)$ & $\begin{array}{r}11400 \\
(1430) \\
\end{array}$ & $12800(1840)$ & $\begin{array}{r}11200 \\
(1530)\end{array}$ \\
\hline Median [Min, Max] & $\begin{array}{c}9500[8250, \\
11500]\end{array}$ & $\begin{array}{c}10900[8090, \\
14600]\end{array}$ & $\begin{array}{l}11100 \\
{[8900,} \\
15500]\end{array}$ & $\begin{array}{c}12400[9940, \\
16900]\end{array}$ & $\begin{array}{c}11100[8090, \\
16900]\end{array}$ \\
\hline
\end{tabular}


Table 2: Association between Shannon alpha diversity of saliva samples and child growth metrics measured at 3-4 years. Each cell shows the coefficient for each exposure's association with the Shannon index as derived from 6 different linear regression models. Each model is named based on the main body mass exposure of interest.

\begin{tabular}{|c|c|c|c|c|c|c|}
\hline & \multicolumn{6}{|c|}{ Dependent variable: Shannon alpha diversity $(95 \% \mathrm{Cl})$} \\
\hline & \multicolumn{6}{|c|}{ Model } \\
\hline & $\begin{array}{c}\text { Crude } \\
\text { TFM }\end{array}$ & $\begin{array}{l}\text { Crude } \\
\text { BMI z- } \\
\text { score }\end{array}$ & $\begin{array}{c}\text { Crude } \\
\text { overweight } \\
\text { status }\end{array}$ & $\begin{array}{c}\text { Adjusted } \\
\text { TFM }\end{array}$ & $\begin{array}{c}\text { Adjusted } \\
\text { BMI z- } \\
\text { score }\end{array}$ & $\begin{array}{l}\text { Adjusted } \\
\text { overweight } \\
\text { status }\end{array}$ \\
\hline $\begin{array}{l}\text { Total fat mass in } \\
\text { kg }\end{array}$ & $\begin{array}{l}-0.041^{*} \\
(-0.084 \\
0.002)\end{array}$ & & & $\begin{array}{l}-0.034 \\
(-0.083 \\
0.015)\end{array}$ & & \\
\hline BMI z-score & & $\begin{array}{l}-0.056^{* *} \\
(-0.110,- \\
0.002)\end{array}$ & & & $\begin{array}{l}-0.060^{*} \\
(-0.119 \\
0.0001)\end{array}$ & \\
\hline Overweight & & & $\begin{array}{l}-0.056 \\
(-0.188 \\
0.075) \\
\end{array}$ & & & $\begin{array}{l}-0.036 \\
(-0.183 \\
0.111) \\
\end{array}$ \\
\hline $\begin{array}{l}\text { Solid foods start } \\
\text { age (months) }\end{array}$ & & & & $\begin{array}{l}-0.007 \\
(-0.058 \\
0.044)\end{array}$ & $\begin{array}{l}-0.008 \\
(-0.059 \\
0.043)\end{array}$ & $\begin{array}{l}-0.007 \\
(-0.059 \\
0.044)\end{array}$ \\
\hline Female & & & & $\begin{array}{l}-0.038 \\
(-0.166 \\
0.089) \\
\end{array}$ & $\begin{array}{l}-0.045 \\
(-0.172 \\
0.081) \\
\end{array}$ & $\begin{array}{l}-0.044 \\
(-0.172 \\
0.084) \\
\end{array}$ \\
\hline $\begin{array}{l}\log _{10-} \\
\text { transformed } \\
\text { sample age in } \\
\text { days }\end{array}$ & & & & $\begin{array}{l}-1.802 \\
(-4.413 \\
0.809)\end{array}$ & $\begin{array}{l}-1.82 \\
(-4.412 \\
0.771)\end{array}$ & $\begin{array}{l}-1.917 \\
(-4.534 \\
0.700)\end{array}$ \\
\hline $\begin{array}{l}\text { Gestational age } \\
\text { (in weeks) }\end{array}$ & & & & $\begin{array}{l}-0.011 \\
(-0.045 \\
0.024)\end{array}$ & $\begin{array}{l}-0.011 \\
(-0.045 \\
0.023)\end{array}$ & $\begin{array}{l}-0.012 \\
(-0.046 \\
0.023)\end{array}$ \\
\hline C-section & & & & $\begin{array}{l}-0.022 \\
(-0.170 \\
0.125)\end{array}$ & $\begin{array}{l}-0.02 \\
(-0.167 \\
0.126)\end{array}$ & $\begin{array}{l}-0.023 \\
(-0.171 \\
0.125)\end{array}$ \\
\hline $\begin{array}{l}\text { Maternal BMI } \\
\left(\mathrm{kg} / \mathrm{m}^{2}\right)\end{array}$ & & & & $\begin{array}{l}0.015^{\star *} \\
(0.003 \\
0.028) \\
\end{array}$ & $\begin{array}{l}0.017^{\star *} \\
(0.004 \\
0.029) \\
\end{array}$ & $\begin{array}{l}0.015^{\star *} \\
(0.002 \\
0.027) \\
\end{array}$ \\
\hline & & & & & & \\
\hline Intercept & $\begin{array}{l}2.954^{* *} \\
(2.717 \\
3.191)\end{array}$ & $\begin{array}{l}2.757^{* *} \\
(2.696 \\
2.818) \\
\end{array}$ & $\begin{array}{l}2.751^{* *} \\
(2.684, \\
2.819)\end{array}$ & $\begin{array}{l}8.682^{* *} \\
(0.589 \\
16.774) \\
\end{array}$ & $\begin{array}{l}8.562^{* *} \\
(0.513 \\
16.611) \\
\end{array}$ & $\begin{array}{l}8.931^{* *} \\
(0.808 \\
17.054) \\
\end{array}$ \\
\hline Observations & 236 & 236 & 236 & 202 & 202 & 202 \\
\hline $\mathbf{R}^{2}$ & 0.014 & 0.017 & 0.003 & 0.055 & 0.064 & 0.047 \\
\hline Adjusted $\mathbf{R}^{2}$ & 0.01 & 0.013 & -0.001 & 0.02 & 0.03 & 0.012 \\
\hline
\end{tabular}




\section{Supplementary Files}

This is a list of supplementary files associated with this preprint. Click to download.

- Supplementaryfigurestablessept10.docx 